\title{
Sedating critically ill patients
}

Ming Hua Cheng ${ }^{1}$, MMed, Tong Khee $\underline{\operatorname{Tan}}^{1}$, FRCA

S edation has allowed doctors to perform procedures that might otherwise be unpleasant and terrifying, thereby deterring patients from accessing them. The ideal pharmacological therapy would provide safe analgesia, hypnosis and anxiolysis, with exemplary pharmacokinetic properties such as organ-independent elimination, predictable distribution and rapid titratability. These qualities would enable rapid recovery to 'street fitness' and even allow procedures to be performed outside traditional hospital settings.

Little evidence is available to suggest the superiority of one agent over another, ${ }^{(1)}$ although synergistic combination therapies are frequently used. Commonly used agents include benzodiazepines, intravenous anaesthetic agents, opioids, neuroleptic agents and centrally acting alpha agonists. Doctors undertaking sedation require detailed knowledge of these agents and how they interact in the context of the patient's medical profile. Misguided use of sedation has resulted in morbidity and death. ${ }^{(2)}$ With increasing sedation depth, patients' ability to maintain cardiovascular stability and independently maintain airway patency and ventilatory function may become impaired, and they may require critical interventions to avoid the possibility of 'lost airway'. Crucially, monitoring of vital signs needs to be delegated to appropriately skilled personnel who can undertake any medical intervention required ${ }^{(3)}$ while the procedurist concentrates on the task at hand.

\section{INTENSIVE CARE UNIT SEDATION}

A lesser-known realm of sedation practices is that of intensive care unit (ICU) sedation, which is a highly specialised and demanding technique since these sedating drugs are used on some of the sickest patients with deranged physiology.

In neurocritical care, sedation is an indispensable therapeutic pillar in which injured brains are 'rested' to minimise brain metabolic activity and as a key therapeutic component for control of raised intracranial pressure. ${ }^{(4)}$ In all ICUs, some critically ill patients are humanely sedated to improve experiences that are probably terrifying and noxious, including tracheal intubation, mechanical ventilation, invasive procedures such as line insertion or tissue sampling, and the necessary routine of orotracheal suctioning. This is advocated by the clinical practice guidelines for pain, agitation, delirium, immobility and sleep disruption. ${ }^{(5)}$

At another extreme, terminal or palliative sedation is sometimes practised in the ICU. The double-edged sword that is ICU sedation results in various central nervous system side effects such as delirium and agitation. ${ }^{(1)}$ These are usually characterised by acute changes in motor and psychological activities, and frequently accompanied by a loss of control of action and disorganisation of thought. It may occur in isolation or be accompanied by other disorders such as severe anxiety and terror. The aetiology of delirium is multifactorial, ranging from the illness process, such as metabolic disorders, sepsis-associated encephalopathy and its associated treatment, to external factors such as noise, discomfort, pain, disorientation and sedation. Agitated and delirious ICU patients are a danger to themselves, as they risk dislodgement of lines and tubes, have increased metabolic requirements and subsequently have a greater risk of dementia and cognitive decline; their medical and nursing care may also be affected. ${ }^{(6)}$ There is also an association with increased cost and length of stay in the ICU. (7) Needless to say, it is distressing for family and friends to witness a loved one in such a state and bound by restraints.

Assessment tools such as the Richmond Agitation and Sedation Scale and the Confusion Assessment Method for the Intensive Care Unit (CAM-ICU) are well validated and frequently used in many ICUs. ${ }^{(8)}$ They allow repeated assessments over monitoring epochs. Familiarity with these tools has allowed for protocols to be written and studies to be designed to better understand the factors contributing to quality sedation and side effects such as delirium.

In this issue of the Singapore Medical Journal, Ng et al investigated sedation practices (i.e. sedation practices in intensive care evaluation [SPICE]) in Singapore public hospital ICUs to overcome the knowledge gap on this topic. ${ }^{(9)}$ Data was compared with that from Malaysia and Australia/New Zealand (ANZ), since a uniform protocol, ANZ SPICE, was used. ${ }^{(10,11)}$ The Singapore data showed lower proportions of ICU patients in the deep sedation range. CAM-ICU-positive delirium (at least one episode) was seen in $23.7 \%$ of their study cohort, far lower than the $44 \%-50.7 \%$ incidence in the other two SPICE studies..$^{(9-1)}$ At the risk of simplifying cause and effect, there was a higher frequency of benzodiazepine use in both the ANZ and Malaysian studies, while propofol and fentanyl were the most commonly used agents locally. Moreover, the local regimen's target was in the range of light sedation. Light sedation has been advantageous for reducing ventilation time when compared with deep sedation, as described by Treggiari et al. ${ }^{(12)}$ Importantly, it also reduces subsequent posttraumatic stress four weeks after hospital discharge.

\section{THE FUTURE OF SEDATION PRACTICES}

The association between sedation and delirium/agitation has directed research towards finding drugs with better sedation profiles, using the best sedation regimen and better-defined,

1Department of Intensive Care, Singapore General Hospital, Singapore

Correspondence: A/Prof Tan Tong Khee, Senior Consultant, Department of Intensive Care, Division of Anaesthesiology, Singapore General Hospital, Outram Road, Singapore 169608. tan.tong.khee@singhealth.com.sg 
individualised sedation goals. ${ }^{(13)}$ Creating or finding the best assessment regimens is also vitally important. These must be practical to be implemented across very heterogeneous ICU settings, as rightly evinced by $\mathrm{Ng}$ et al. ${ }^{(9)}$

Strategies to decrease overall exposure to sedation, ${ }^{(14)}$ such as sedation hold/interruption, have failed to show benefit in terms of reducing the duration of mechanical ventilation or ICU stay, according to a study by the SLEAP Investigators and Canadian Critical Care Trials Group. ${ }^{(15)}$ Current practices have moved away from continuous deep sedation, and with more suitable agents available, ICU patients will hopefully be at less risk of developing the morbidity associated with agitation and delirium. ${ }^{(7)}$

Protocolised ICU management ${ }^{(16)}$ for analgesia, sedation and delirium, and early use of sedation algorithms and pathways that apply the concept of early goal-directed sedation, as reported by the recently completed SPICE III, ${ }^{(17)}$ have also been shown to support clinical decision-making and improve clinical outcomes (e.g. subsyndromal delirium and mortality). Moreover, SPICE III demonstrated laudatory properties of the use of dexmedetomidine. ${ }^{(17)}$ MIND-USA ${ }^{(18)}$ (antipsychotics for delirium and sedation sparing effect) and MENDS II ${ }^{(19)}$ (studying sedative choice in severe sepsis patients to best reduce delirium, improve survival and long-term brain function) are examples of studies that will continue to seek even better sedation for the critically ill. These ongoing studies will hopefully guide intensivists on their choice of sedating agents and adjuncts in order to achieve physiological endpoints with the least haemodynamic and mental perturbations.

The future of sedation is exciting. Picture the interplay of novel sedatives and the use of target-controlled infusion algorithms, which are informed by pharmacokinetic and pharmacodynamic principles. These will be integrated with the patient's physiological monitors, including depth of sedation monitoring. Such a system will react appropriately and automatically to clinical requirements while at all times keeping the patient safely sedated via feedback loops. Each component is currently being used in various aspects of clinical practice. However, a skilled human is still needed to orchestrate this complex task through the act of titration. Hence, more studies, such as the study by $\mathrm{Ng}$ et $\mathrm{al}^{\left({ }^{(9)}\right.}$ are needed to focus interest and resources and to further develop cutting-edge sedation practices and technology in our local ICUs.

\section{REFERENCES}

1. Hughes CG, McGrane S, Pandharipande PP. Sedation in the intensive care setting. Clin Pharmacol 2012; 4:53-63.

2. Amornyotin S. Sedation-related complications in gastrointestinal endoscopy. World J Gastrointest Endosc 2013; 5:527-33.

3. Wong Meng Hang v. Singapore Medical Council [2018] SGHC 253.

4. Durrmeyer X, Vutskits L, Anand KJ, Rimensberger PC. Use of analgesic and sedative drugs in the NICU: integrating clinical trials and laboratory data. Pediatr Res 2010; 67:117-27

5. Devlin JW, Skrobik Y, Gélinas C, et al. Clinical practice guidelines for the prevention and management of pain, agitation/sedation, delirium, immobility, and sleep disruption in adult patients in the ICU. Crit Care Med 2018; 46:e825-73.

6. Ouimet S, Kavanagh BP, Gottfried SB, Skrobik Y. Incidence, risk factors and consequences of ICU delirium. Intensive Care Med 2007; 33:66-73.

7. Shehabi Y, Bellomo R, Mehta S, Riker R, Takala J. Intensive care sedation: the past, present and the future. Crit Care 2013; 17:322.

8. Ely EW, Inouye SK, Bernard GR, et al. Delirium in mechanically ventilated patients: validity and reliability of the confusion assessment method for the intensive care unit (CAM-ICU). JAMA 2001; 286:2703-10.

9. Ng SY, Phua J, Wong YL, et al. Singapore SPICE: sedation practices in intensive care evaluation in Singapore-a prospective cohort study of the public healthcare system. Singapore Med J 2020: 61:19-23.

10. Shehabi Y, Bellomo R, Reade MC, et al; Sedation Practice in Intensive Care Evaluation (SPICE) Study Investigators; ANZICS Clinical Trials Group. Early intensive care sedation predicts long-term mortality in ventilated critically ill patients. Am J Respir Crit Care Med 2012; 186:724-31.

11. Shehabi Y, Chan L, Kadiman S, et al; Sedation Practice in Intensive Care Evaluation (SPICE) Study Group Investigators. Sedation depth and long-term mortality in mechanically ventilated critically ill adults: a prospective longitudinal multicentre cohort study. Intensive Care Med 2013; 39:910-8.

12. Treggiari MM, Romand JA, Yanez ND, et al. Randomized trial of light versus deep sedation on mental health after critical illness. Crit Care Med 2009; 37:2527-34.

13. Young J, Murthy L, Westby M, Akunne A, O'Mahony R; Guideline Development Group. Diagnosis, prevention, and management of delirium: summary of NICE guidance. BMJ 2010; 341:c3704

14. Dale CR, Kannas DA, Fan VS, et al. Improved analgesia, sedation, and delirium protocol associated with decreased duration of delirium and mechanical ventilation. Ann Am Thorac Soc 2014; 11:367-74

15. Mehta S, Burry L, Cook D, et al; SLEAP Investigators; Canadian Critical Care Trials Group. Daily sedation interruption in mechanically ventilated critically ill patients cared for with a sedation protocol: a randomized controlled trial. JAMA 2012; 308:1985-92.

16. Skrobik Y, Ahern S, Leblanc M, et al. Protocolized intensive care unit management of analgesia, sedation, and delirium improves analgesia and subsyndromal delirium rates. Anesth Analg 2010; 111:451-63.

17. Shehabi Y, Howe BD, Bellomo R, et al; ANZICS Clinical Trials Group and the SPICE III Investigators. Early sedation with dexmedetomidine in critically ill patients. N Engl J Med 2019; 380:2506-17.

18. Girard TD, Exline MC, Carson SS, et al; MIND-USA Investigators. Haloperidol and ziprasidone for treatment of delirium in critical illness. N Engl J Med 2018; 379:2506-16.

19. Maximizing the efficacy of sedation and reducing neurological dysfunction and mortality in septic patients with acute respiratory failure (MENDS2). In: ClinicalTrials.gov [online]. Available at: https://clinicaltrials.gov/ct2/show/ NCT01739933. Accessed August 19, 2019. 